\title{
Numerical Optimization of Hybrid Dielectric/HTS Resonators for Surface Impedance Evaluation of HTS Films
}

\author{
C. Collado, D. Gonzalo, E. Rozan, J.M. O’Callaghan. \\ Universitat Politecnica de Catalunya. Campus Nord UPC-D3. Barcelona 08034, Spain. \\ C. Sans \\ Universitat de Vic. C/ Miramarges 4. Vic 08500, Spain
}

\begin{abstract}
This work describes an alternative to the traditional dielectric resonator topology used for measuring surface impedance in High Temperature Superconducting (HTS) films. A gap is introduced above the dielectric so that only the lower film is in direct contact with it. This arrangement has been used extensively for mechanical tuning of dielectric resonators and, when used for surface impedance measurement, it can be designed to make the losses in the upper film small rellative to the overall resonator losses. Then, measured results are mostly due to one of the films and not the average of two. The specifics of a resonator design for measuring 2-inch wafers are presented. An analysis and optimization of the resonator is done using a numerically efficient mode-matching algorithm.
\end{abstract}

\section{INTRODUCTION}

Commonly used techniques for evaluating surface impedance in HTS thin films are based on measurements of resonators on either patterned, planar structures or in a dielectric resonator cavity in which the upper and lower walls are made of HTS films and are in contact with the dielectric [1]. Only the latter approach is non-destructive, but it yields the average surface resistance of two films. Thus, to measure a sample with unknown properties, it has to be paired with a reference sample to determine its surface impedance. The degree of accuracy to which the surface resistance of the reference is known can be a significant source of error in these measurements. Therefore, other non-destructive methods need to be explored further.

\section{HYBRID DIELECTRIC/HTS RESONATOR}

The dielectric resonator topology under study is a modification of the traditional dielectric-HTS one [1], where a gap is introduced between the upper endplate and the dielectric cylinder (Fig. 1). The purpose of introducing this gap is to lower the RF losses in the upper film and

Manuscript received September 15, 1998

This work has been funded by the Spanish Ministry of Education and Culture through CICYT grant MAT95-1038-C02-02 and by the Catalan CIRIT through grant SGR00043.

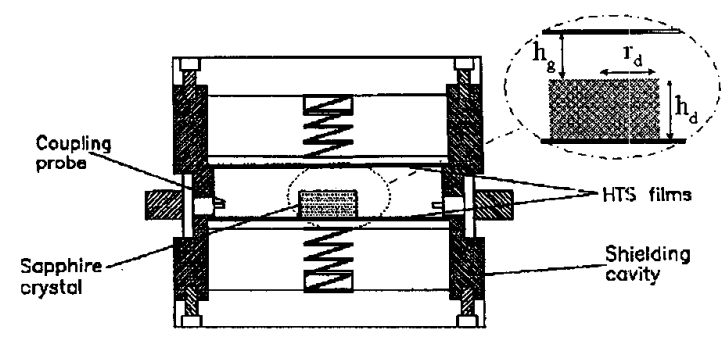

Fig. 1. Dielectric resonator structure. Definitions of dielectric height $\left(h_{d}\right)$, dielectric radius $\left(r_{d}\right)$ and gap size $\left(h_{\mathcal{g}}\right)$

concentrate them in the lower one, so that its $R_{s}$ is the largest contributing factor to the total losses of the cavity.

Experimental use of this type of cavity for measurement of $R_{s}$ is mentioned in [2], but no design details are given. An analytical study of this topology, based on an axial modematching method with a single mode is presented in [3]. No description is given in any of these works of the procedure to optimize the cavity for $R_{s}$ determination purposes. In [3] it is pointed out that, as the upper gap is increased, the electromagnetic fields on the normal metal side walls also increase, so the contribution of the RF losses of the upper film are reduced at the expense of increasing those of the side walls. For $R_{s}$ determination, an optimal height exists for a given resonator size and a given diameter of the superconducting endplates, for which the ratio of the RF losses in the lower plate to the overall losses in the cavity is maximum. This optimum may be found using an efficient mode-matching code [4], which calculates the electromagnetic field distribution in structures with azimutal symmetry. The algorithm is not restricted to small gap sizes and can be used to optimize the whole structure (gap size, dielectric height and dielectric diameter) for a given diameter of the supeconducting wafers to be measured.

Reference [2] proposes a surface resistance determination procedure based in two consecutive measurements of quality factors in which the top and bottom samples are exchanged. The optimization proposed here might make this unnecessary, so that a single measurement and a rough estimate of the surface resistance of the top film and side walls might lead to an accurate determination of the surface resistance of the lower film. 


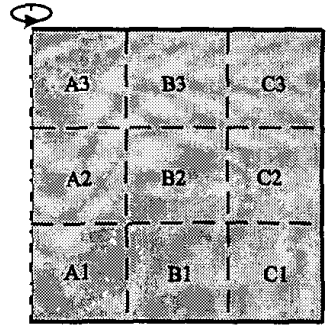

Fig. 2. Radial regions $(A, B, C)$ and axial layers $(1,2,3)$ considered by the mode-matching algorithm. Dielectric permitivity can be defined independently for each of the 9 cells (A1..C3).

\section{A. Full-wave radial mode-matching method}

The software developed [4] can analyze up to three different radial regions, each one divided into three different layers (Fig.2). The dielectric constant in each region and layer can be set independently, and the whole volume analyzed is assumed to be enclosed by conducting walls. Fields in every radial region are expressed as an infinite series of particular solutions of the wave equation in cylindrical coordinates.

Azimutal and axial eigenfunctions are harmonic functions while radial eigenfunctions are Bessel functions, which must be chosen appropriately to avoid singularity in the axis (region A), fulfill boundary conditions on the external walls (region $\mathrm{C}$ ), or provide continuity between adjacent regions (region B). The eigenvalues of each radial region are found by solving a pair of transcendental equations related to the three-layer problem. A system of equations is then found by forcing continuity conditions of the tangential fields between radial regions $A$ and $B$, and $B$ and $C$, from which the coefficients of the mode expansion are found. In our case 12 modes are sufficient. Fig. 3 illustrates the field and current distributions for a sapphire dielectric with a $12 \mathrm{~mm}$ diameter and a $6 \mathrm{~mm}$ height.

\section{B. Optimization. Basics and alternatives}

For $R_{s}$ determination, the optimal dimensions of the cavity in Fig. 1 are those that make the losses of the lower plate dominant with respect to the other type of losses. This is equivalent to maximizing $Q_{o} / Q_{\text {low }}$, where:

$Q_{0}=\frac{\omega_{0} U}{P_{d}} ; \quad Q_{l o w}=\frac{\omega_{0} U}{P_{d_{-} l o w}}$,

$U$ is the total stored energy in the cavity, $P_{d}$ the total dissipated power, $P_{d \_l o w}$ the power dissipated in the lower wall, and $Q_{0}$ the unloaded quality factor. Both the total energy $U$ and the dissipated powers $P_{d}$ and $P_{d_{-} \text {low }}$ can be calculated from the field distribution inside the cavity with properly defined surface and volume integrals of the electromagnetic fields [5]. Definitions analogous to that of $Q_{l o w}$ in (1) can be used for $Q_{u p}, Q_{s d}$ and $Q_{d}$ which account for the losses in the upper wall, side wall, and dielectric respectively. Thus, the unloaded quality factor can be written as:
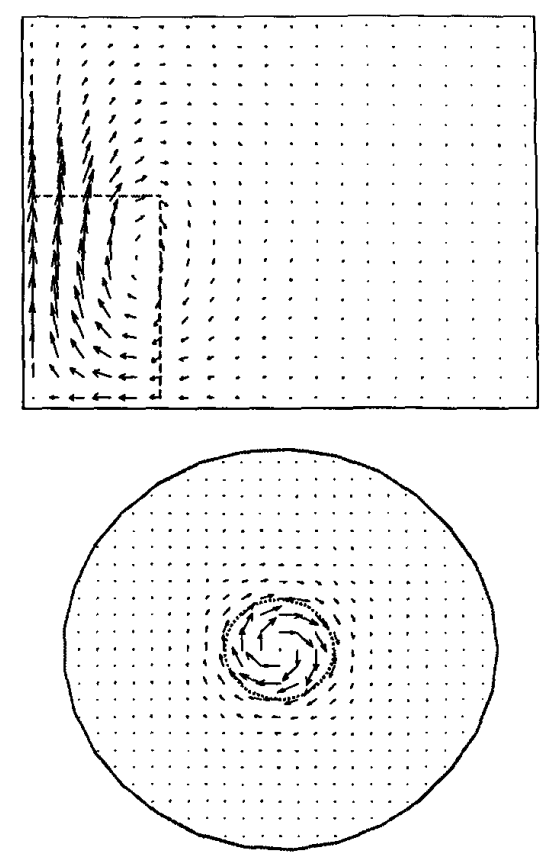

Fig. 3. Field and current distributions in the cavity with a $\mathrm{TE}_{0}$ mode and a sapphire dielectric. Bottom plate diameter is $48 \mathrm{~mm}$, dielectric diameter is $12 \mathrm{~mm}$ and dielectric height is $6 \mathrm{~mm}$. The dashed line represents the sapphire outline. (Top) Radial cut of the resonator showing magnetic field lines. (Bottom) Currents at the surface of the lower HTS film.

$\frac{1}{Q_{0}}=\frac{1}{Q_{l o w}}+\frac{1}{Q_{u p}}+\frac{1}{Q_{s d}}+\frac{1}{Q_{d}}$

The relevance of maximizing $Q_{0} / Q_{l o w}$ is due to the fact that this defines the sensitivity of the system:

$\left|\frac{\Delta Q_{0} / Q_{0}}{\Delta R_{s} / R_{s}}\right|=\frac{Q_{0}}{Q_{\text {low }}}$,

which can be proven from (1) and (2), and from the proportionality between $P_{d_{-} l o w}$ and $R_{s}$. Also, as $Q_{0} / Q_{\text {low }}$ is increased, the accuracy in estimating $Q_{u p}, Q_{s d}$ and $Q_{d}$ has a lesser effect on the $R_{s}$ predicted from the measured $Q_{0}$ and (2).

A software code has been developed, which includes the electromagnetic mode-matching algorithm described previously, and is capable of calculating $Q_{l o w}, Q_{u p}, Q_{s d}$ and $Q_{d}$ as a function of dielectric height $\left(h_{l}\right)$, dielectric radius $\left(r_{d}\right)$ and gap size $\left(h_{g}\right)$ (Fig. 1). To test it, the cavity dimensions given in [2] were used, and the code results were compared with those in Table $I$ in that reference. The agreement is excellent, as shown in Table I of this work.

The software developed can use several strategies to vary $h_{d}, r_{d}$ and $h_{g}$ in the search for a maximum of $Q_{0} / Q_{l o w}: 1$ ) variation of the gap size for a given (fixed) dimension of the dielectric; 2) exhaustive search varying $h_{d}, r_{d}$ and $h_{g}$ over a specified range, with a specified incremental step size; and 3 ) 
TABLE I

COMPARISONS BETWEEN [2] AND THIS WORK

Glow is defined as the product of the surface resistance of the lower plate and Qlow; Gup and Gsd are defined similarly.

\begin{tabular}{lcccc}
\hline & $\mathrm{f}(\mathrm{GHz})$ & Glow & Gup & Gsd \\
\hline$[2]$ & 9.5 & 546 & $4.90 \mathrm{e} 3$ & $1.15 \mathrm{e} 6$ \\
This work & 9.510 & 541 & $4.81 \mathrm{e} 3$ & $1.11 \mathrm{e} 6$ \\
Error \% & & $0.8 \%$ & $1.7 \%$ & $3.3 \%$ \\
\hline
\end{tabular}

gradient search using Matlab [6]. In this process it is assumed that the upper and lower walls are made of $\mathrm{YBa}_{2} \mathrm{Cu}_{3} \mathrm{O}_{7-\delta}$ (YBCO), and the side walls of a normal metal (usually OFHC copper, but it can be changed by the user). Their $R_{s}$ is scaled as the calculated resonant frequency of the cavity changes due to variations in $h_{d}, r_{d}$ and $h_{g}$. The scaling law is taken as $f^{2}$ for YBCO and $f^{I / 2}$ for the metal, and the values of $R_{s}$ at $10 \mathrm{GHz}$ and $77 \mathrm{~K}$ are taken as $300 \mu \Omega(\mathrm{YBCO})$ and $9 \mathrm{~m} \Omega(\mathrm{Cu})$ [7] respectively. The frequency dependence of the loss tangent of sapphire is neglected, taking $\tan \delta=410^{-8}$ [8] throughout the optimization.

\section{Optimum gap for fixed dielectric and wafer sizes}

When dimensions of the wafer and the dielectric are known and fixed, only $h_{g}$ needs to be varied to find a maximum for $Q_{o} / Q_{\text {low }}$. This is done for two reasons: to explore a possible improved design of the cavity in [2] (while maintaining the sapphire used); and to find a good initial set of values of $h_{d}, r_{d}$ and $h_{g}$ from which a second optimization can be done using a gradient search in which all three variables are allowed to change.

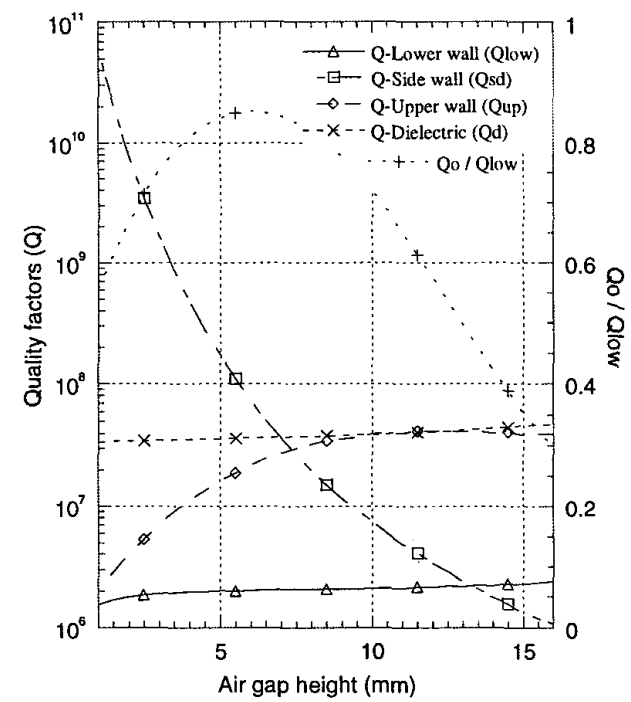

Fig. 4. Dependence of $Q_{u p}, Q_{l v w}, Q_{s d}$ with gap height $\left(h_{g}\right)$. The optimum height (max. $Q_{o} / Q_{\text {low }}$ ) represents a trade-off between the losses in the upper and side walls $\left(Q_{u p}, Q_{s t}\right)$, whose dependence on $h_{g}$ is much stronger than that of the lower wall $\left(Q_{l o w}\right)$ and dielectric $\left(Q_{d}\right)$. The maximum of $Q_{0} / Q_{\text {low }}$ is at $h_{g}=6 \mathrm{~mm}$.
Fig. 4 shows the results of the gap height optimization for a dielectric with $h_{d}=6 \mathrm{~mm}, r_{l}=6 \mathrm{~mm}$ and a 2 inch wafer where only an area of $48 \mathrm{~mm}$ in diameter is exposed to the electromagnetic fields in the cavity. This figure indicates that, while the losses in the side walls are strongly dependent on $h_{g}$, this dependence is moderate in the upper wall, and weak in the dielectric and lower wall. Therefore, the optimum height represents a trade-off between the losses in the upper wall and those in the side wall. This results in an optimum gap of $h_{g}=6 \mathrm{~mm}$ and $Q_{0} / Q_{l o w}=0.85$, which essentially coincides with the results reported in [2]. As shown later, substantial improvements can be made by adjusting $h_{d}, r_{d}$ and $h_{g}$ jointly.

Finally, Fig. 5 shows that these results degrade strongly if the diameter of metal side wall is reduced.

\section{Substitution of the upper HTS plate by a cooper one}

Unsuccessful attempts have been made at replacing the YBCO in the upper wall by copper. Even when the gap height optimization process was performed taking into account the new $R_{s}$ of the upper plate, the sensitivity $\left(Q_{0} / Q_{\text {low }}\right)$ values were too low to allow an acceptable accuracy in the determination of the $R_{s}$ of the bottom plate. In other words, the gap can never be high enough to replace the upper film by a normal metal without making it a dominant. source of loss in the cavity and rendering it useless for surface resistance determination. Fig. 6 shows the effects of this substitution in the sensitivity $\left(Q_{0} / Q_{l o w}\right)$.

\section{E. Joint optimization of $h_{d}, r_{d}$ and $h_{g}$}

The values of $h_{d}, r_{d}$ and $h_{g}$ have been optimized using two parallel approaches: a gradient optimization and an

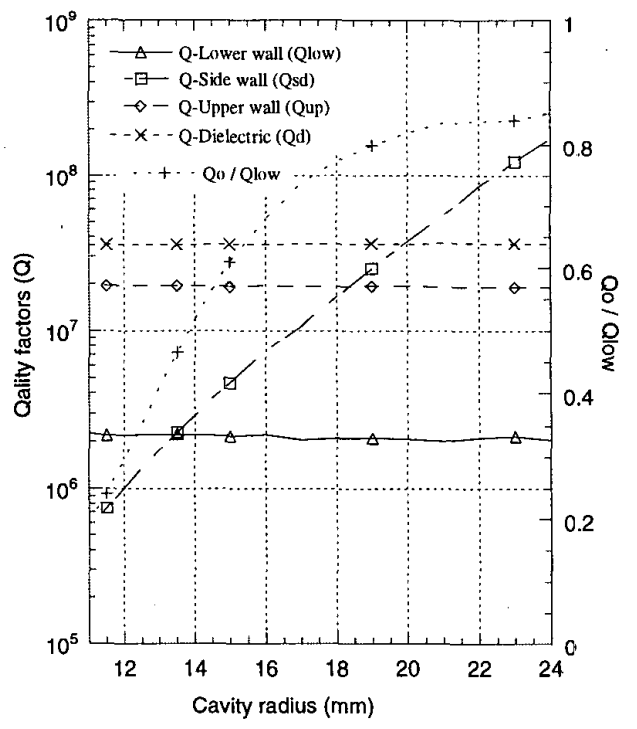

Fig. 5. Dependence of $Q_{u p}, Q_{l o w}, Q_{s d}$ with cavity radius. As the radius is decreased, losses in the side walls increase, become dominant, and degrade $Q_{0} / Q_{\text {low. }}$ Gap height is $h_{g}=6 \mathrm{~mm}$. As in Fig. 4, dielectric height is $h_{d}=6 \mathrm{~mm}$ 


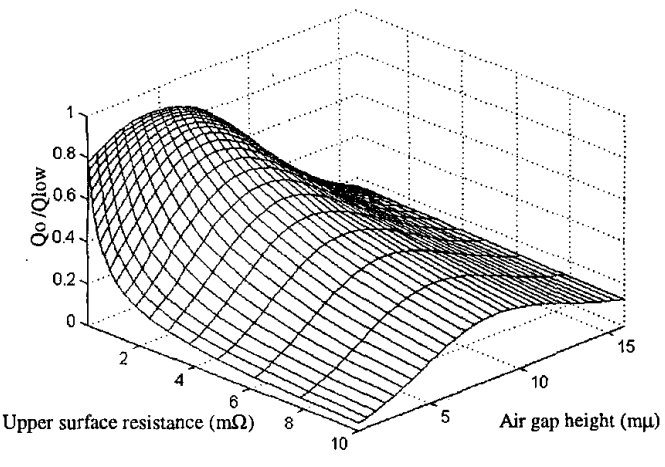

Fig. 6. Deterioration of the sensitivity $\left(Q_{0} / Q_{l o w}\right)$ as the value of $R_{s}$ of the upper sample is increased. A strong degradation occurs for the range of $R_{s}$ values corresponding to cooper at $77 \mathrm{~K}$ at the resonant frequencies of the cavity (about $10 \mathrm{~m} \Omega$ ). For these values of $R_{s}$ in the upper wall, the $Q_{0} / Q_{\text {low }}$ is too low for determination of the $R_{s}$ of the lower HTS film.

exhaustive search over a broad range of $h_{d}, r_{d}$ and $h_{g}$ to ensure that the gradient optimization did not stop at a local maximum of $Q_{0} / Q_{l o w}$.

The exhaustive search was done from $2 \mathrm{~mm}<h_{d}<10 \mathrm{~mm}$, $3 \mathrm{~mm}<r_{d}<14 \mathrm{~mm}$ and $1 \mathrm{~mm}<h_{g}<15 \mathrm{~mm}$. A maximum was found at $h_{l}=2 \mathrm{~mm}, r_{d}=5 \mathrm{~mm}$ and $h_{g}=6 \mathrm{~mm}$ with $Q_{o} / Q_{l o w}=0.94$. The search showed that this optimum value would degrade sharply when $h_{d}$ was decreased beyond its optimum value. However, the dependence with $h_{d}$ and $r_{d}$ was much weaker.

Gradient optimizations were run starting from several values, including the ones from Sect. II-C. They all reached similar values of $Q_{0} / Q_{\text {low }}$. The best one was the one started from $h_{d}=2 \mathrm{~mm}, r_{d}=5 \mathrm{~mm}$ and $h_{g}=6 \mathrm{~mm}$ (the optimum of the exhaustive search), which was used to fine-tune the previous search resulting in $h_{d}=1.7 \mathrm{~mm}, r_{d}=5.0 \mathrm{~mm}$ and $h_{g}=4.6 \mathrm{~mm}$ with $Q_{0} / Q_{\text {low }}=0.95$. In other words, if no other losses are considered in a cavity with these dimensions, only a $5 \%$ overestimation of the surface resistance of the lower sample will be made, and this may be greatly with estimates of the surface resistance of the side walls, upper film and dielectric loss tangent.

\section{CONCLUSION}

An optimization of the image dielectric resonator cavity has been done by numerical techniques. The software developed for the optimization has been cross-checked with previously published results. The optimized cavity should be allow the determination of surface resistance of YBCO with an error lower than $5 \%$ if no other sources of RF loss in the cavity are taken into account.

\section{REFERENCES}

[1] Z-Y Shen, C. Wilker, P. Pang, W. L. Holstein, D. Face, D.J. Konutz. "High Tc Superconductor-Sapphire Microwave Resonator with Extremely High Q-Values up to $90 \mathrm{~K} "$. IEEE Trans. Microwave Theory Tech., vol. 40 no. 12, pp 2424-2432. Dec. 1992.

[2] C. Zuccaro, N. Klein, A. G. Zaitsev, R. Wördenweber, Y. Lemaitre, and J.C. Mage. "Nonlinear microwave losses of large area YBCO thin films. Applied Superconductivity 1997, Inst. Phys. Conf. Ser. no 158 pp 295-298 [Proceedings EUCAS 1997].

[3] N. Tellman, N. Klein, U. Dähnem, H. Schulz, and H. Chaloupka. "High Q $\mathrm{LaALO}_{3}$ dielectric resonator shielded by YBCO films". IEEE Trans. Appl. Superconduct., vol 4, no. 3, pp143-148, Sept. 1994.

[4] Sans, J.M. O'Callaghan, D. Sancho, R. Pous, J. Fontcuberta, J.-F. Liang and G.C. Liang. "Full-wave analysis of the image hybrid dielectric/HTS resonator". IEEE Trans. Appl. Superconduct., vol 4, no. 7, pp3840-3844, Dec. 1997.

[5] R.F. Harrington. Time-Harmonic Electromagnetic Fields. McGrawHill, 1961.

[6] The Mathworks, Inc. Matlab user's guide.

[7] G.W.C. Kaye and T.H. Laby. Table of physical and Chemical constants. Longmans Green London, 1966.

[8] Z.-Y. Shen. High Temperature Superconducting Microwave Circuits. Artech House, 1994. 